\title{
3D Space-Time Visualization of Player Behaviour in Pervasive Location-Based Games
}

\author{
Paul Coulton, Will Bamford, Keith Cheverst, and Omer Rashid \\ Infolab21, Lancaster University, Lancaster, LA1 4WA, UK \\ Correspondence should be addressed to Paul Coulton, p.coulton@lancaster.ac.uk
}

Received 21 January 2008; Accepted 17 April 2008

Recommended by Andrew Davison

Location-based games not only offer new experiences for the players, but also present new challenges for researchers in terms of analyzing player behaviour. Whilst many ethnographical studies have presented useful qualitative insights into this area, there is the potential to both improve support for these studies and to provide more effective representations of the quantitative data that can often be extracted from the game itself in a manner that enables greater understanding. In this paper, we illustrate how combined spatial and temporal information can be represented using the human geographers' technique of space-time paths to provide 3D visualizations of a player's or players' movement. Our analysis of a particular location-based game shows how a richer understanding of overall game play is obtained and highlights the possibilities for using the technique for a whole range of locationbased services to provide a more complete view of complexities of journeys. Further, we discuss how these techniques can be utilized more generally by ethnographers who study the behaviour of mobile actors.

Copyright (C) 2008 Paul Coulton et al. This is an open access article distributed under the Creative Commons Attribution License, which permits unrestricted use, distribution, and reproduction in any medium, provided the original work is properly cited.

\section{INTRODUCTION}

Location-based gaming allows users to play games that incorporate knowledge of their physical location and landscape, and then provides them with the ability to interact with both real and virtual objects within that space. These games are often created to answer various research questions, not only for games researchers but also for those interested in related topics such as technologies for ubiquitous computing platforms, human computer interaction, or collaborative behaviour. In terms of analyzing these games, various techniques have been used to provide both qualitative and quantitative analysis. Ethnographical techniques have been used successfully [1] to provide insights into how users interact, both with each other and the game space, for information that is often not explicitly represented in the game events recorded in the system, such as a discussion of tactics or the verbal directions from an online game controller [2]. Benford et al. highlighted in 2002 that "there was no agreed format for logging or suites of analyzing tools" [3] for studying system data quantitatively, and in the years since little seems to have changed. Further, in a recent paper by Crabtree et al. [4] they defined both the need for tools and their required characteristics to better support ethnographical study of pervasive and ubiquitous systems particularly in mobile environments. This is not to say tools have not been considered and, indeed, in the aforementioned paper by Benford et al., they discuss using an indexed 3D recording of a performance that can be replayed and analyzed. Whilst this would provide a useful system, it requires a great deal of computational support and for some games that do not incorporate a complex virtual world, such as those that are principally mobile phone based, it is difficult to implement. Other less complex 2D systems [3,5] have also been proposed but, as with the previous system, they principally provide a replay of the action at instances in time rather than an overall perspective on the spatial and temporal movement of the players in the game. In order to enable this perspective, in this paper we highlight the use of space-time paths which were originally proposed by the Swedish geographer Hägerstrand in 1970 [6] as a means of quantitatively analyzing human migration patterns.

A space-time path illustrates how a person navigates their way through the spatial-temporal environment. The physical area is reduced to a two-dimensional plane with these dimensions representing a person's top-down position (e.g., longitude and latitude). Their location and destination are represented as zero-dimensional points. Time is represented 


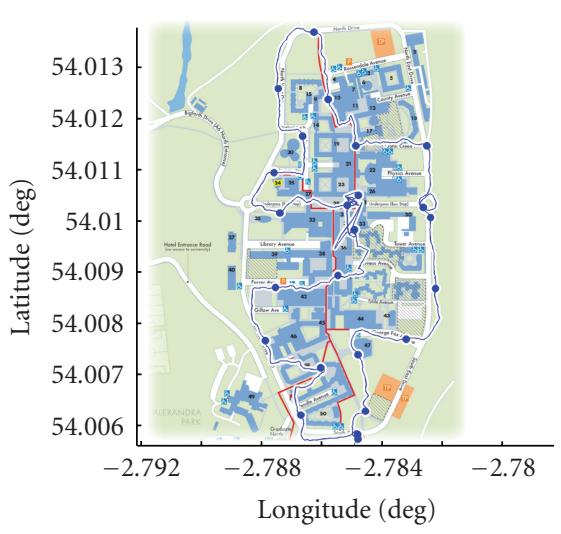

(a)

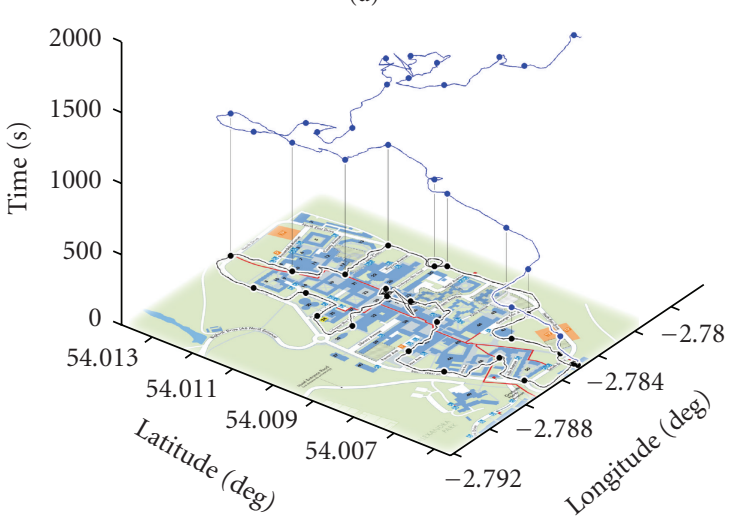

(b)

FIgURE 1: 2D path and 3D space-time path of a journey around Lancaster University campus (7th of April 2007).

by the vertical axis. These three dimensions combine to form a world representing a specific portion of space-time. The path of a stationary individual will appear as a vertical line between the starting and ending times at a specific location. If an individual moves between two geographical locations at a constant velocity, the graph will show a line with a fixed trajectory which joins the start and end points. The slower an individual travels, the greater the line's vertical gradient. Therefore, vertical gradient of the plot is inversely proportional to the user's ground-based velocity.

In Figure 1, we illustrate both a 2D spatial plot and a 3D space-time path for an actor walking around the campus at Lancaster University-the data was collected at one-second intervals using a modified version of Locoblog [7]. It is evident in this figure that whilst the $2 \mathrm{D}$ plot provides us with details of the particular locations visited, it provides no specific information relating to the actor's movement along the path itself, in other words it provides information about the route and nothing about the particulars of the journey. The view that the journey itself should form part of the study of mobility has recently gained popularity amongst sociologists studying transportation and is transforming the view that the time on the move should be considered as "lost" and of less relevance than the locations themselves [8].

Representing spatial and temporal data and its relationship to cognitive processes is beyond the scope of this paper.

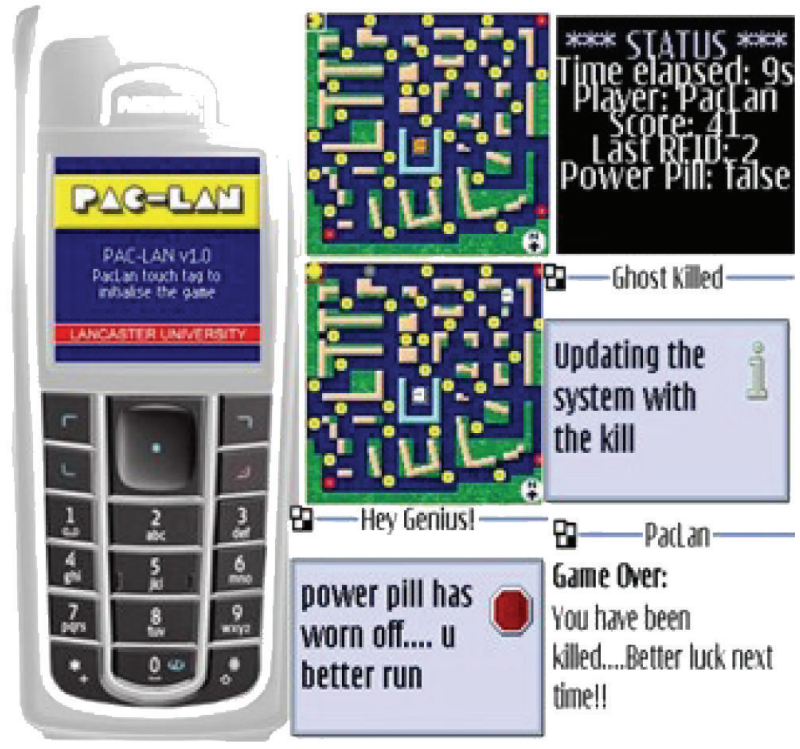

Figure 2: PAC-LAN client.

However, by observing the $3 \mathrm{D}$ space-time path shown in Figure 1, we believe it provides a greater sense of the actor's movement and a clearer picture of the direction of travel, the speed of movement, and any places, where the actors paused or delayed their travel.

Although the space-time path does not provide the fine detail of a particular journey, it could be annotated with data from an ethnographical study, such as photos, audio, video, and text from the user themselves to produce a more holistic representation. In this paper, we will illustrate how space-time paths can be used as means of analyzing player behaviour for the mobile mixed-reality locationbased game PAC-LAN [9]. However, this technique can be applied generically across a variety of location-based applications irrespective of the location sensing technology or the underlying communications.

\section{PAC-LAN}

PAC-LAN is a novel version of the classic video game Pac-Man, in which human players use the Alexandra Park accommodation complex at Lancaster University as the game maze [9]. The player who takes the role of the main PACLAN character collects game pills (using a Nokia 5140 mobile phone equipped with a Nokia Xpress-on RFID reader shell) which are in the form of yellow plastic discs fitted with adhesive RFID tags placed around the maze. Four other players take the role of the "Ghosts" (Mr. Blue, Mr. Red, Mr. Orange, and Mr. Pink) who attempt to hunt down the PACLAN player.

The mobile phone game client is implemented on the Java 2 microedition (J2ME) platform which is connected to a central server using a general packet radio service (GPRS) connection. The server relays to the PAC-LAN character client his/her current position along with the position of all Ghosts based on the pills collected as shown in Figure 2. 


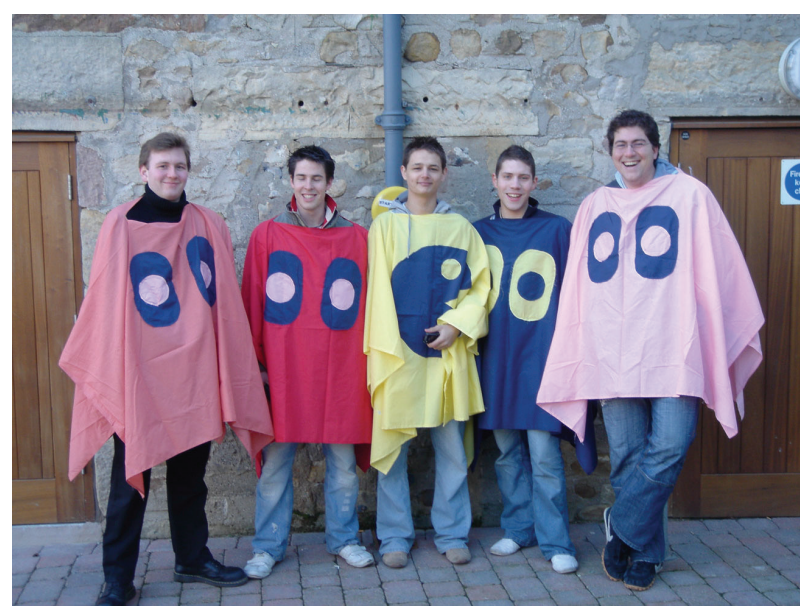

Figure 3: PAC-LAN trial team for 10th of February.

The game pills are used by the Ghosts to obtain the PACLAN character's last-known position and to reset their kill timer which, if expired, will prevent them from "killing" PAC-LAN. In this way, the ghosts must regularly interact with the server which is then able to relay their position to PAC-LAN. The Ghosts can "kill" the PAC-LAN character by detecting him/her via an RFID tag fitted to their costume (assuming their kill timer has not expired). Once PAC-LAN is killed, the game is over and the points for the game are determined by calculating the number of game pills collected and time taken to do so. When PAC-LAN tags one of the red power pills, indicated by all Ghost icons changing to white on the phone screen, he/she is then able to kill the Ghosts, and thus gain extra points, using the same RFID detection process. "Dead" Ghosts must return to the central point of the game maze where they can be reactivated into the game.

It should be noted that PAC-LAN uses an implied, rather than measured, method of location positioning [2] in that the players' positions are obtained through their interaction with an object (game pill) at a known location.

\section{ANALYZING PLAYER BEHAVIOUR}

To illustrate the use of space-time paths in location-based game analysis, we will not try to encompass the numerous games of PAC-LAN which have been played thus far [9] but rather consider a specific game which took place on the morning of 10th of February 2006. The players, in this case, were five males from Lancaster University between the ages of 22 and 27 shown in Figure 3.

None of the players had previous experience of the game nor had any of them used an RFID-enabled mobile phone. Furthermore, only Mr. Pink was familiar with the game's real-world environment (he lived in the accommodation area which forms the game's maze). The game in question was shorter than average at 16 minutes and 30 seconds (the average game duration was 26 minutes), although the action was fairly typical of a first play experience.

In terms of mapping the game, we have created a Java class that uses the JBDC connector library to link the database of our game server to Matlab. This method not only provides a quick and easy method for producing the space-time paths, but also allows us to generate them in realtime as the game progresses. Once the paths are generated, Matlab allows us to manipulate them in 3D space, as shown in Figure 4, which is useful for extracting detail and allows various combinations of players to be selected.

In all the Figures used for the game analysis of PACLAN, we have not represented the axes of the spatial plane in meters or degrees and minutes but rather in terms of the pixels as they would appear on the phone screen map. For example, the actual game maze area is approximately 350 meters squared and the mobile phone map of this maze is represented as a $128 \times 128$ pixel bitmap. Therefore, each pixel edge represents a length of approximately 2.74 meters.

Figure 4 illustrates that at the start of the game, the path taken by Mr. Blue bears relatively little correlation to that of PAC-LAN but as the game progresses it can clearly be observed that Mr. Blue starts to track the movements of PACLAN.

In Figure 5, we show the space-time paths of all four Ghosts together with that of PAC-LAN. It is worth noting that unlike other location-based implementations of PacMan, such as Human Pac-Man [4] or Pac-Manhattan (www. Pac-Manhattan.com), there are no online controllers and each player acts on his/her own initiative within the game [9].

From this, we can see those space-time paths of Mr. Blue appear to closely correlate with PAC-LAN, whilst Mr. Orange's path is similar but with greater divergence at more points. Conversely, Mr. Red generally appears to have run around fairly aimlessly, as Mr. Pink does although he confined himself to a much smaller spatial area.

To ascertain the effectiveness of the space-time path, consider Figure 6 which presents a running correlation of the paths taken by each of the Ghosts relative to that of the PAC-LAN players. From this figure, it can be seen that the paths taken by Mr. Blue and Mr. Pink are more closely correlated than either Mr. Orange or Mr. Red. However, from the previous analysis of the space-time paths it was observed that Mr. Pink's path was less correlated to PAC-LAN. This apparent discrepancy is due to the fact that the correlation merely provides a simple distance measure and because Mr. Pink remained fairly central in the game maze he maintained a good correlation.

This actual player activity can best be illustrated through Figure 7 which shows each player's movement as a circle whose radius is the mean displacement centered around his/her average position to give some sense of how adventurous each player was during the game. Whilst this gives a view of general activity it does not provide the detail of the space-time path.

Our observations during the game and the video footage confirm what was observed in the space-time paths. This is not to say that we should rely on purely quantitative analysis, in fact, the qualitative observations and discussions revealed that Mr. Red was not as aimless in his movements as it may at first have appeared; rather, he claimed his primary strategy during the game was to ambush PAC-LAN, and his patterns 


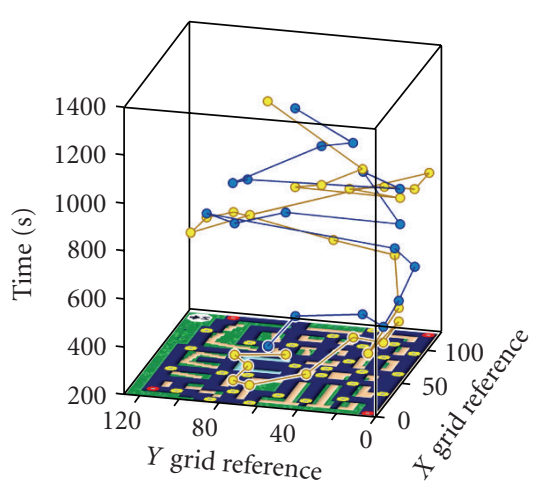

(a)

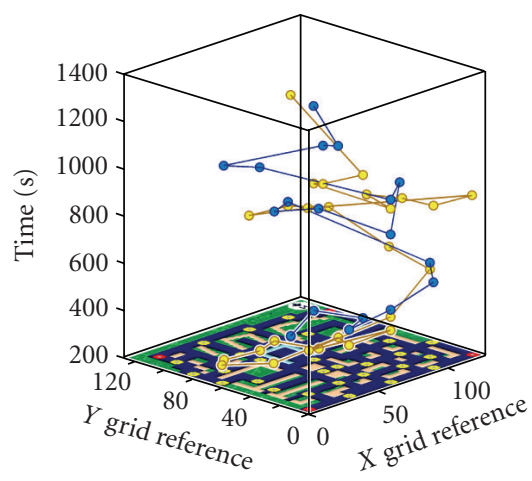

(c)

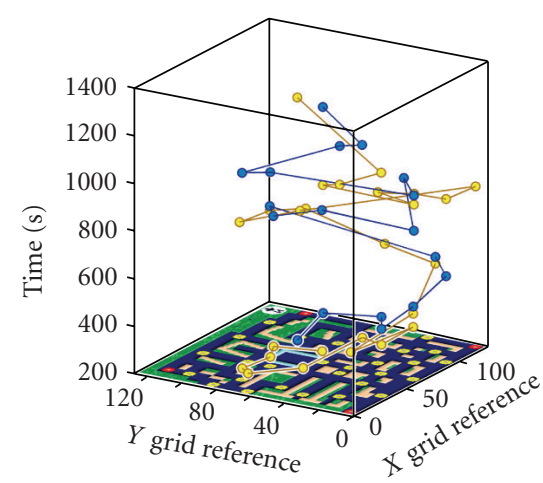

(b)

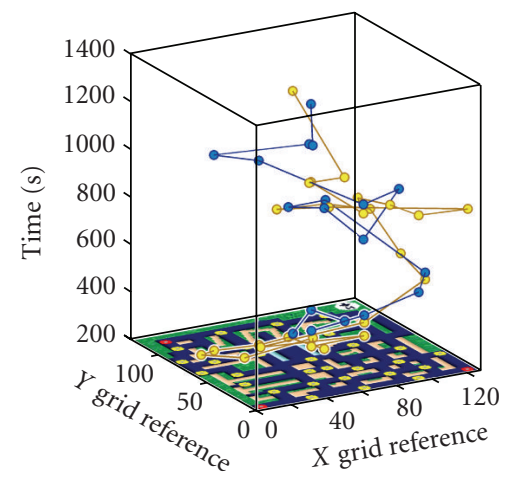

(d)

FIgURE 4: Rotating space-time paths for PAC-LAN and Mr. Blue.

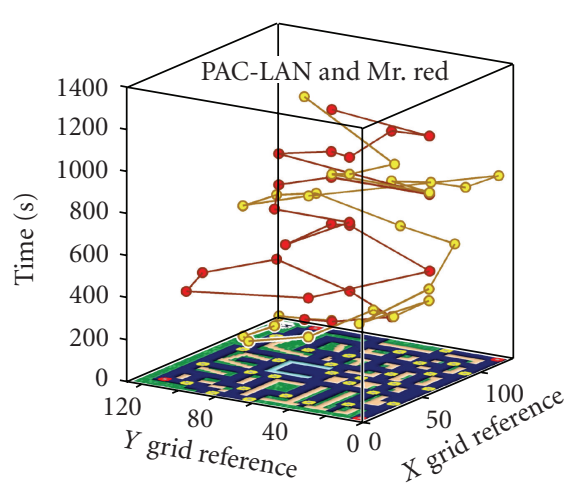

(a)

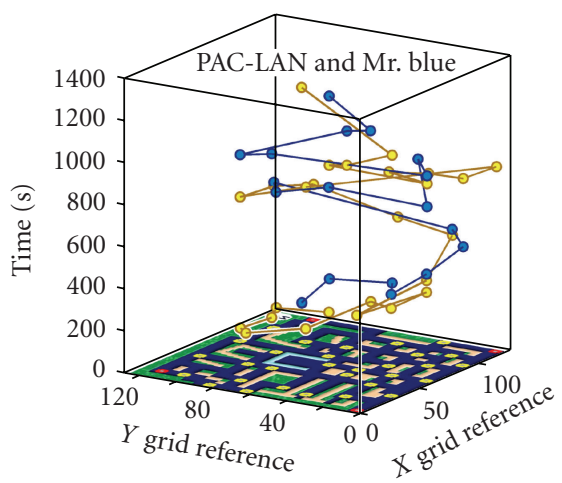

(c)

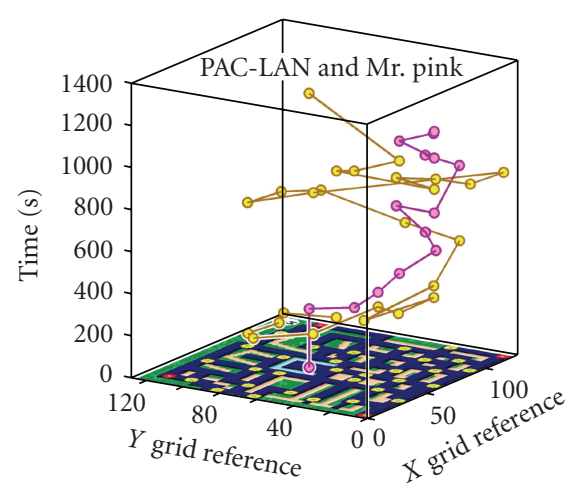

(b)

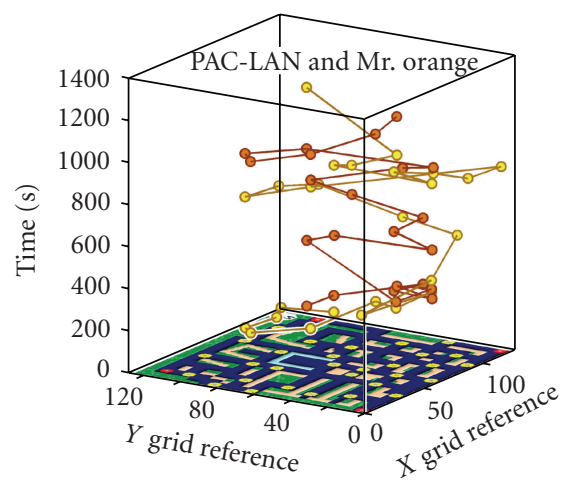

(d)

FIGURE 5: Space-time paths of PAC-LAN compared to each of the four Ghosts. 


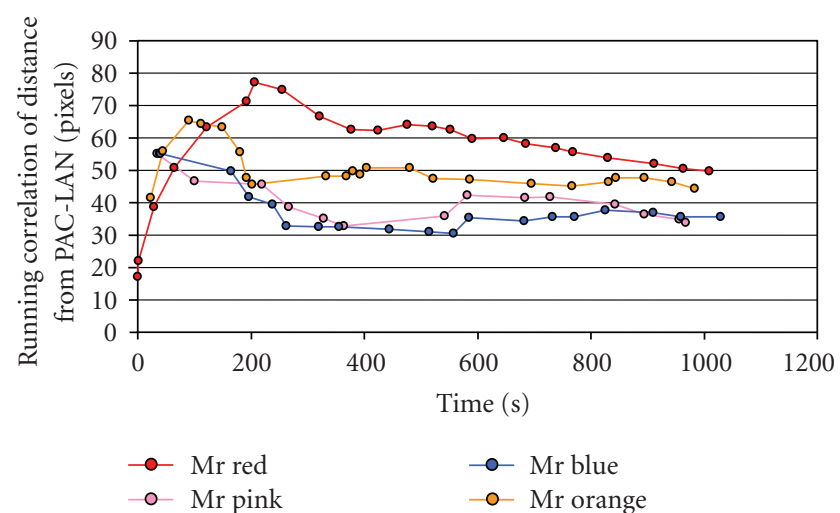

FIgURE 6: Running correlation of the Ghosts' paths through the game relative to that of PAC-LAN.

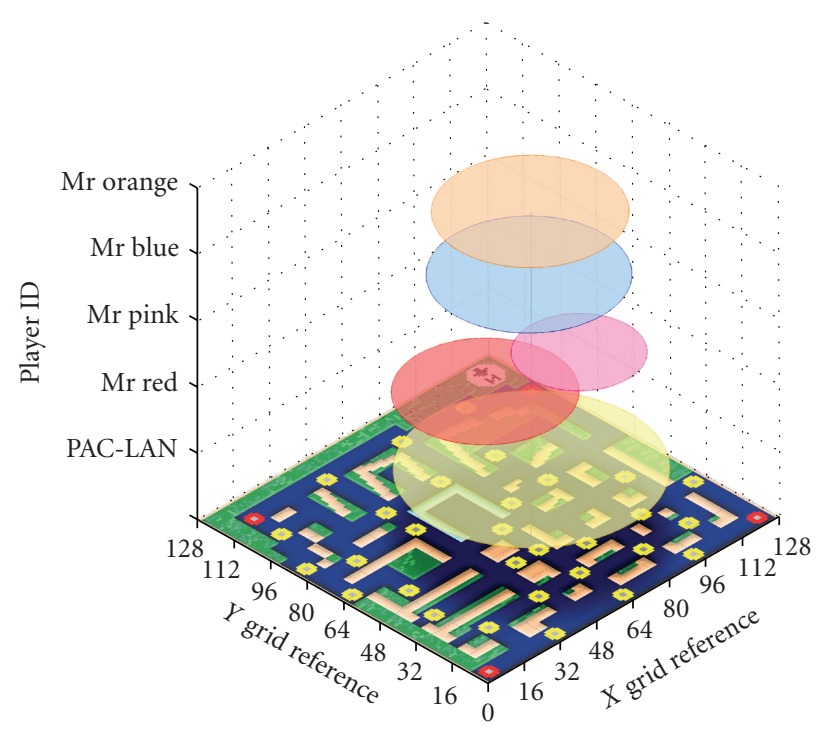

FIGURE 7: Players' mean displacement from their averaged position.

of movement reflect this (PAC-LAN was indeed caught in such a maneuver).

\section{CONCLUSIONS}

In this paper, we have presented human geographers' space-time paths as an effective means of analyzing player behaviour in location-based games. We believe that this technique has significant potential for representing actors' spatial and temporal movements in many location-based scenarios, and, if coupled with qualitative data, can provide significant advantages when analyzing emerging computing environments. We are already working with sociologists and geographers to create versions of Locoblog that will aid them in ethnographic studies, and with the forthcoming visualization tool it will provide significant opportunities for behavioural analysis of mobile actors. In respect of meeting the requirement of tools to be used in ethnographical study, we believe it addresses many of the more salient points identified by Crabtree and Rouncefield [1] of capture, extraction, synchronization, and representation. The current generation of mobile phones is capable of recording text, image, video, and voice, and it also provides an effective means of capture when coupled with an application such as Locoblog. By recording the data to the phone card in standard formats, it can then be easily extracted and combined as required. In terms of synchronization, the GPS unit provides a universal timestamp with which we can synchronize the often multiple sources of mobile information and this time is available even when there are insufficient satellites to provide a positional fix. Finally, in terms of representation, the space-time map presents a very powerful method of representing combined spatial and temporal behaviour.

\section{ACKNOWLEDGMENT}

The authors would like to express their thanks to Nokia for the provision of software and hardware to the Mobile Radicals research group at Lancaster University which was used for the implementation of this project.

\section{REFERENCES}

[1] A. Crabtree and M. Rouncefield, "Working with text logs: some early experiences of e-social science in the field," in Proceedings of the 1st International Conference on eSocial Science, Manchester, UK, June 2005.

[2] O. Rashid, I. Mullins, P. Coulton, and R. Edwards, "Extending cyberspace: location based games using cellular phones," Computers in Entertainment, vol. 4, no. 1, pp. 3-20, 2006.

[3] S. Benford, M. Fraser, G. Reynard, B. Koleva, and A. Drozd, "Staging and evaluating public performances as an approach to CVE research," in Proceedings of the 4th International Conference on Collaborative Virtual Environments (CVE '02), pp. 80-87, Bonn, Germany, September-October 2002.

[4] A. Crabtree, S. Benford, C. Greenhalgh, P. Tennent, M. Chalmers, and B. Brown, "Supporting ethnographic studies of ubiquitous computing in the wild," in Proceedings of the Conference on Designing Interactive Systems: Processes, Practices, Methods, and Techniques (DIS '06), pp. 60-69, University Park, $\mathrm{Pa}$, USA, June 2006.

[5] N. Nova, F. Girardin, G. Molinari, and P. Dillenbourg, "The underwhelming effects of automatic location-awareness on collaboration in a pervasive game," in Proceedings of the International Conference on the Design of Cooperative Systems, Carry-le-Rouet, Provence, France, May 2006.

[6] T. Hägerstrand, "What about people in regional science?" Papers in Regional Science, vol. 24, no. 1, pp. 6-21, 1970.

[7] W. Bamford, P. Coulton, and R. Edwards, "Space-time travel blogging using a mobile phone," in Proceedings of the 4th International Conference on Advances in Computer Entertainment Technology (ACE '07), vol. 203, pp. 1-8, Salzburg, France, June 2007.

[8] C. Licoppe and Y. Inada, "Emergent uses of a multiplayer location-aware mobile game: the interactional consequences of mediated encounters," Mobilities, vol. 1, no. 1, pp. 39-61, 2006.

[9] O. Rashid, W. Bamford, P. Coulton, R. Edwards, and J. Scheible, "PAC-LAN: mixed-reality gaming with RFID-enabled mobile phones," Computers in Entertainment, vol. 4, no. 4, 2006. 

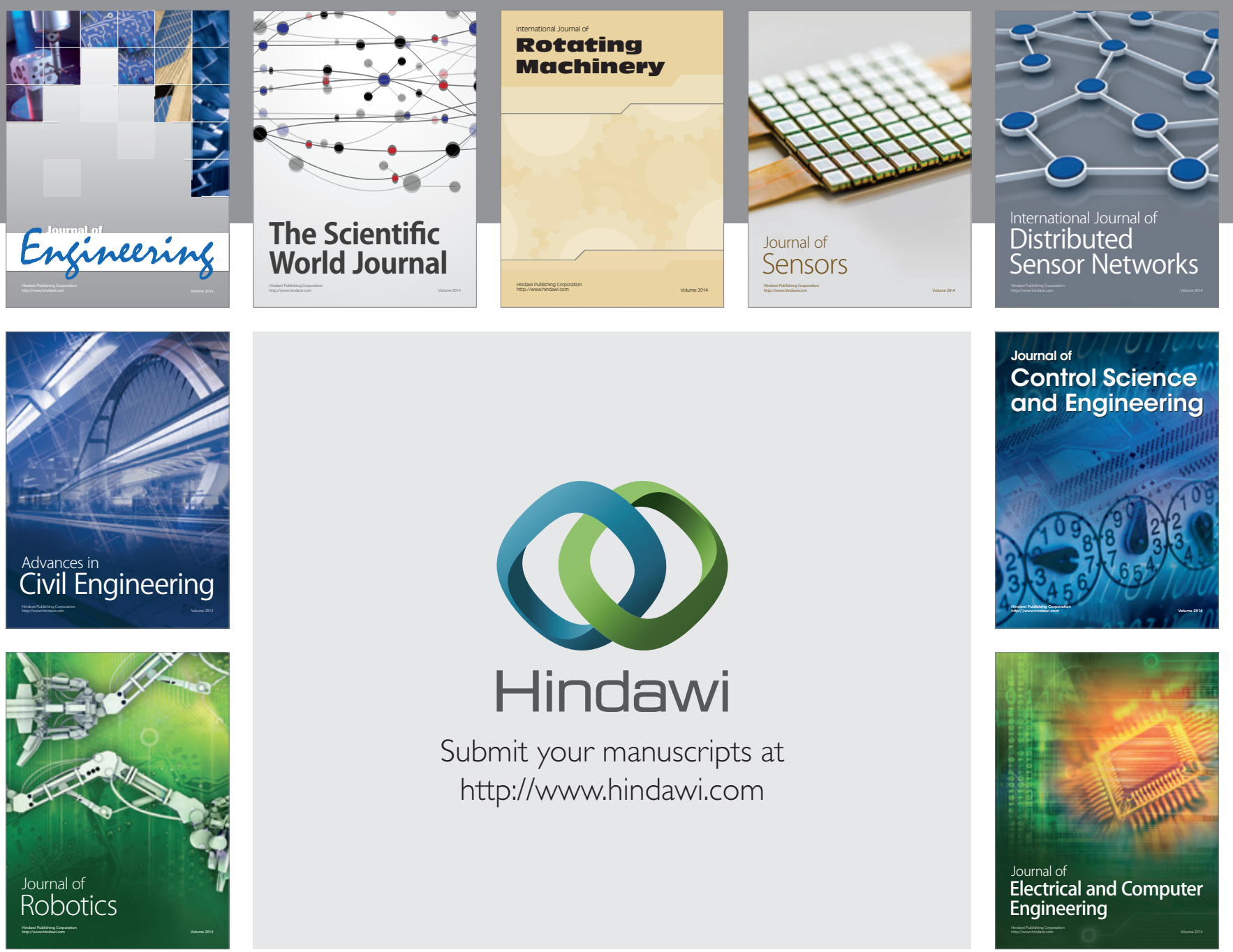

Submit your manuscripts at

http://www.hindawi.com
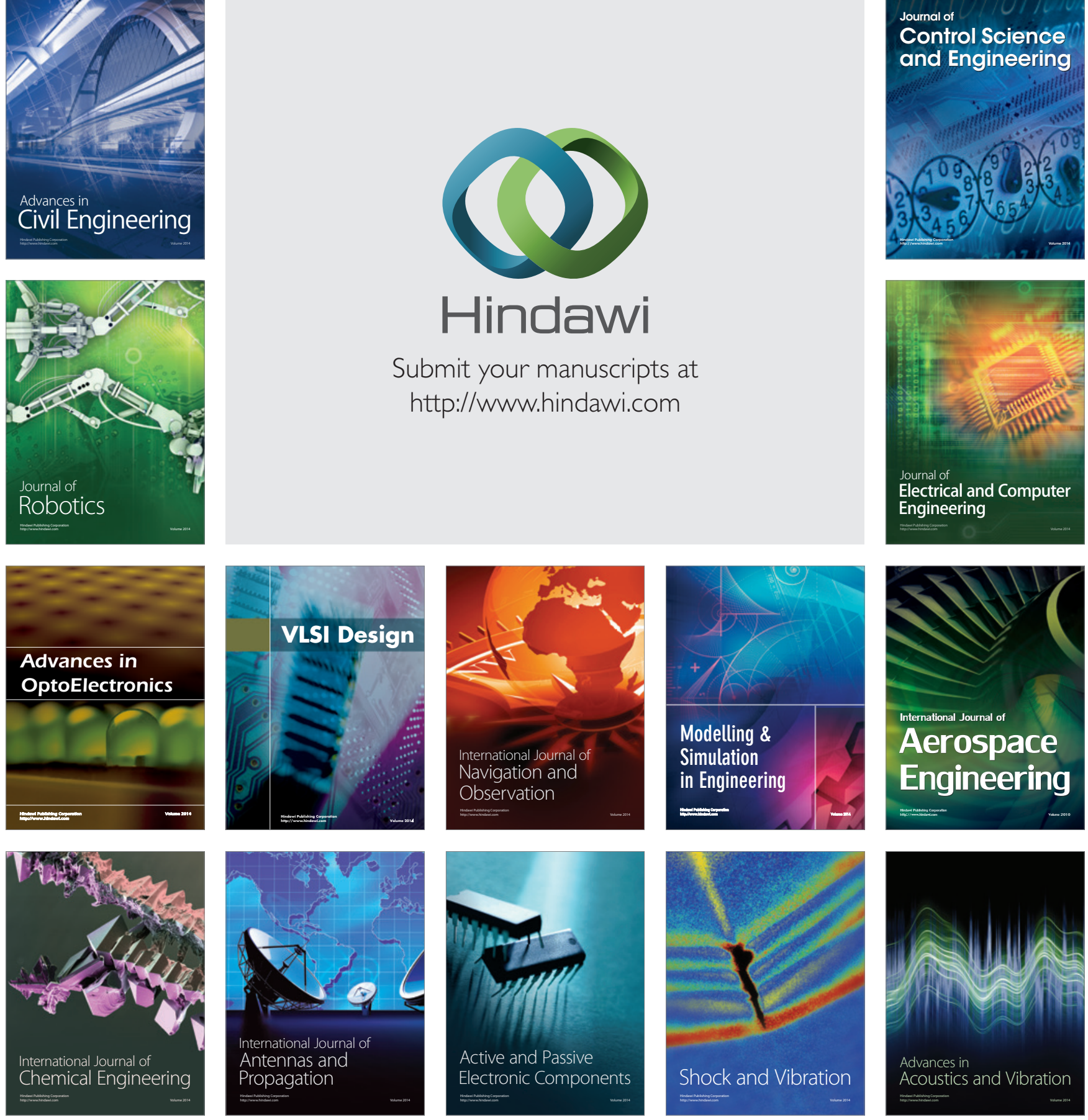